\title{
Percepciones sobre la política de seguridad democrática de Colombia en la frontera Táchira-Norte de Santander (2002-2010)
}

Ana Marleny Bustamante

\section{RESUMEN}

El presente artículo evalúa los cambios en la percepción sobre la política de seguridad democrática de Colombia (PSD) y su efecto en la seguridad de la frontera de Táchira y Norte de Santander (TNS) durante el período 20022010. Siguiendo la revisión teórica desde las disciplinas que confluyen en el análisis de la seguridad de las poblaciones fronterizas, su interrelación con el entorno local, nacional e internacional se realizó una investigación de tipo cualitativo con entrevistas a informantes para conocer sus percepciones y contrastarlas a la luz de la teoría y los constructos emergentes de la información cualitativa. Los resultados muestran la emergencia de tres categorías principales: Ambivalencia sobre la capacidad del Estado, oscilaciones en la credibilidad del Estado y pérdida de la calidad de vida. Dichas categorías se subdividen en dimensiones como fortalecimiento y debilidad del Estado,

* Mi agradecimiento al Consejo de Desarrollo Científico Humanístico, Tecnológico y de las Artes (CDCHTA) de la Universidad de Los Andes, Venezuela, por su financiamiento parcial al proyecto código: NUTA-H-400-16-09-B cuyo título es igual al de este artículo. También agradezco grandemente al doctor Douglas Izarra por la revisión metodológica y los comentarios a un primer borrador de este artículo y a Elkin Calles por el apoyo durante la etapa de entrevistas y trabajo de campo.

** Doctora en ciencia política de la Universidad de Liverpool, Visiting Fulbright Scholar en la Universidad Estatal de San Diego. Profesora titular adscrita al Centro de Estudios de Fronteras e Integración (CEFi) de la Universidad de Los Andes (Venezuela) [apernia@ula.ve; apernia5@hotmail.com]; [https://orcid.org/0000-0003-3914-7088].

Recibido: 27 de mayo de 2020 / Modificado: 22 de octubre de 2020 / Aceptado: 10 de diciembre de 2020

Para citar este artículo:

Bustamante, A.M. (2021). Percepciones sobre la política de seguridad democrática de Colombia en la frontera Táchira - Norte de Santander (2002-2010). OASIS, 34, pp. 39-59

DoI: https://doi.org/10.18601/16577558.n34.04 
violencia, éxodo poblacional, desempleo, falta de acompañamiento con políticas de desarrollo, pérdida de libertades y seguridad pública y resiliencia, entre otras. En su conjunto evidencian que la PSD fortaleció relativamente la capacidad del Estado aunque aún necesita rescatar y construir institucionalidad y cambió percepciones sobre las dinámicas de seguridad fronteriza y de la vida en la frontera de TNS. Una evaluación más apropiada requiere mayor investigación a más largo plazo con el fin de evitar los matices y pasiones propios del momento; por lo tanto, es necesario continuar profundizando y evaluando tendencias sobre la PSD en las fronteras de Colombia.

Palabras claves: Política de seguridad democrática, frontera, percepciones, Táchira, Norte de Santander.

\section{PERCEPCTIONS ABOUT THE DEMOCRATIC SECUIRITY POLICY OF COLOMBIA IN THE BORDER TACHIRA-NORTE DE SANTANDER (2002-2010) T2}

\section{ABSTRACT}

This article evaluates the changes in the perception about the Democratic Security Policy of Colombia (PSD) and its effect on the security at the border between Tachira y Norte de Santander (TNS) during 2002-2010. Following the theoretical revision from the disciplines that focus on analyzing security and border studies and their local, regional, national and international perspectives, a qualitative research was carried out by interviewing informers on both sides of the border in order to know their perspectives and compare them with the literature.
The results show the emergence of three main categories: ambivalences about the capacity of the State, oscillations on the credibility of the State and the worsening of the quality of life. These categories are divided up into dimensions such as strengthening and weakening of the Sate, violence, population exodus, unemployment, lack of State support concerning development policies and loss of liberties and public security, among others. In all, the categories show that PSD strengthened the State capacity but it still needs to rescue and build institutionality. It also changed perceptions about border security and life dynamics at the TNS border. A more appropriate evaluation demands more research, with a longer time frame in order to avoid current complexities. Therefore, it is important to continue deepening and evaluating roads of research about the PDD at the Colombian borders.

Keywords: Democratic Security Policy, border, perceptions, Táchira, Norte de Santander.

\section{INTRODUCCIÓN}

El presidente Álvaro Uribe de Colombia (2002-2010) tuvo como eje principal de su política y acción (doméstica e internacional) de gobierno la política de seguridad democrática (PSD), la cual comprendió dos fases: 1. Formulación y 2. Consolidación, y que se denominó Política de Consolidación de la Seguridad Democrática (PCSD). El énfasis estuvo en la desestructuración de las Fuerzas Armadas Revolucionarias de Colombia (Farc) (Granada, Restrepo y Vargas, 2009), y que se articuló con la guerra contra el terrorismo 
iniciada globalmente por Estados Unidos a partir del 11 de septiembre de 2001 ya que se consideraba a Colombia una amenaza para la seguridad regional debido a la evidente debilidad del Estado para controlar su territorio y ser reconocido como el detentador legítimo de poder.

La PSD partió del Plan Colombia del presidente Andrés Pastrana, luego del revés de los Diálogos de Paz y la zona de despeje de San Vicente del Caguán para terminar el conflicto armado (Pizarro, 2003; Russell, 2003). La diferencia sustancial fue la inclusión de la guerra contra el terrorismo y redirigir el combate de la droga hacia los grupos armados. El presidente Uribe modificó el Plan Colombia y orientó la PSD al combate de grupos insurgentes a los que calificó de terroristas, para alinear la política exterior colombiana a la de los Estados Unidos (Pizarro, 2003; Monroy y Ochoa, 2009).

El 2002 fue el año de máxima violencia en Colombia luego del recrudecimiento de 1996, cuando las ofensivas guerrilleras y los grupos paramilitares, agrupados en las Autodefensas Unidas de Colombia (AUC), la desplegaron contra la población civil. Sin embargo, el 2002 también fue el inicio del declive estratégico de las organizaciones guerrilleras Farc y Ejército de Liberación Nacional, ELN, tanto en expansión territorial como en el desarrollo de sus planes y proyectos (Sánchez y Díaz, 2007).

El triunfo de Uribe en 2002 evidenció el agotamiento del pueblo colombiano con la guerrilla como actor político y confiable, y con la lucha armada entre la guerrilla y las AUC que dejaban de ser percibidas como una lucha de $\mathrm{y}$ en las regiones periféricas para convertirse en algo más cercano e importante debido a que afectaba las vías de comunicación entre las ciudades más importantes y los centros de producción económicos (González, 2006). Más adelante, Uribe logra reelegirse en 2006 a partir de la exhibición del control territorial y debilitamiento de la guerrilla.

La PSD y la PCSD, desplegadas entre 2002 y 2010, incluyeron reformas en las fuerzas armadas, el crecimiento y transformación del aparato de seguridad y defensa del Estado colombiano, un reacomodo de los grupos violentos, la recuperación de las principales carreteras y ciudades y la reducción de la capacidad operacional de los grupos insurgentes, en especial las Farc y las AUC; lo que produjo un punto de quiebre del conflicto (Granada, Restrepo y Vargas, 2009), al punto de llevar al Ministerio de la Defensa a señalar que se estaba en la última etapa del conflicto.

Si bien es cierto que la PSD le ganó la confianza doméstica al replegar los conflictos internos a zonas periféricas y la alianza de los Estados Unidos evidenciada en la ayuda financiera, apoyo logístico y militar al gobierno del presidente Uribe (Cardozo, 2010), no tuvo igual éxito con sus vecinos: Ecuador y Venezuela, países de los cuales dependía para lograr contener a los grupos irregulares (Tickner y Pardo, 2003) en las fronteras comunes, los cuales se veían obligados al repliegue a la periferia o hacia la frontera. Colombia contaba con este apoyo para reducir aún más y eliminar la capacidad operativa de los grupos irregulares en conflicto.

Venezuela y Colombia fueron antagonistas en las visiones sobre el desarrollo, la economía y sociedad o la política exterior. La PSD, así como la proximidad colombiana a Estados 
Unidos, fueron criticadas y rechazadas por el gobierno venezolano, que no solo se opuso enfática y comunicacionalmente, sino que produjo fuertes desencuentros bilaterales: 1 . La firma de un tratado de libre comercio entre Colombia y Estados Unidos, que luego se usó como argumento para justificar el retiro de la Comunidad Andina, 2. El bombardeo a las bases de las Farc en Ecuador y la muerte del máximo dirigente de las Farc Raúl Reyes, desencadenó una fuerte crisis diplomática con amenazas bélicas y, 3. El uso de las bases aéreas por parte de Estados Unidos en territorio colombiano y el posible desbalance armamentista entre países, entre otros. $\mathrm{Al}$ respecto, Cardozo (2010) señaló que políticamente "la construcción de la alianza Colombia-EE.uU. luce tan fuerte como la erosión y aumento de tensiones entre Venezuela - Colombia y EE.UU." (p. 10).

Estos desencuentros binacionales causaron un impacto en la dinámica y vida fronteriza a la cual se le agrega una creciente inseguridad pública (Morffe, 2014). Se destaca la ralentización de lo institucional, visible en las comisiones binacionales que trataban asuntos fronterizos: la Comisión Binacional Fronteriza (Combifron) sobre asuntos de seguridad fronteriza dejó de funcionar en el 2002 y la Comisión de Integración y Asuntos Fronterizos (Copiaf) que venía trabajando desde 1989 y que se reunía semestralmente, dejó de reunirse en 2007 (Cardozo, 2011).

Así, la necesidad de cooperación de los países vecinos de la PSD conflictúa con las de sus visiones de país y con sus políticas exteriores; dejando a las fronteras y sus habitantes en el medio, sin capacidad de acción indepen- diente y con creciente inseguridad pública. Concentrar la seguridad en lo militar (Fuerzas Armadas Nacionales), dejó en un muy lejano segundo plano el aparato de resguardo, control, vigilancia y seguimiento policial que requiere la vida pública y ciudadana (Morffe, 2014).

En razón a lo expuesto se busca responder preguntas tales como: ¿Cuáles son los cambios que se perciben en la dinámica fronteriza derivados de la puesta en práctica de la política de seguridad democrática y de la política de consolidación de la seguridad democrática de Colombia?, ¿pueden atribuirse estos cambios a la militarización de la política de seguridad fronteriza de Colombia?, ¿cuáles han sido los grupos más afectados de las comunidades locales por estos cambios?, ¿cómo se han adaptado los miembros de las comunidades fronterizas a la realidad cambiante en la frontera?, ¿en qué medida los desencuentros en las políticas de los gobiernos de Colombia y Venezuela durante el período 2002-2010 han afectado la percepción de la frontera y del otro país?

El artículo consta de 5 partes principales: La primera se dedica a la presentación del problema de investigación, seguido de la revisión de la literatura desde las perspectivas de los estudios de seguridad en las fronteras para incluir el de seguridad fronteriza. La tercera parte señala la metodología seguida, mientras que la cuarta se dedica a la presentación de los resultados para, finalmente, en la quinta parte se interpretan y analizan los resultados, conclusiones y aportes al área del conocimiento, así como las posibles líneas a seguir para continuar avanzando en el conocimiento del tema de la seguridad fronteriza. 


\section{REVISIÓN DE LA LITERATURA}

La literatura sobre fronteras ha dado paso a conceptos y estudios que parten de la población que habita en esos lugares (Martínez, 1994; Grimson, 2000; García Canclini, 2000; Paasi, 1999; Kolossov, 2005; Newman, 2012; Kolossov y Scott, 2013; Bressan, 2016). Si bien es cierto que el referente principal sigue siendo el Estado-nación, la realidad del creciente poblamiento de las fronteras internacionales obliga a considerarlos como tema de estudio particular por las distintas disciplinas de las ciencias sociales en donde a los conceptos tradicionales es obligante añadir al límite internacional, la cooperación y los intercambios transfronterizos de los pobladores de la frontera como factores intervinientes. Ello a pesar de los avances y retrocesos de procesos y tendencias globales como la globalización y la integración regional.

Desde la perspectiva de los estudios sobre fronteras, antes del 11/09 el mundo estaba racionalizando, en términos de políticas y acciones, el desmembramiento de la Unión Soviética, la aceleración de la integración regional y del proceso de globalización (Newman, 1999). Esta racionalización había llevado a pensar que los límites serían más simbólicos que reales en el sentido que las personas y los bienes se movían y moverían con creciente libertad a través de los límites y las fronteras (Paasi, 1999) y que las poblaciones asentadas en estos espacios tendrían una función más relevante en la construcción de nuevas identidades y en el diseño de las políticas internacionales de los Estados. Adicionalmente, y desde diferentes perspectivas metodológicas, los estudios sobre fronteras, incluyendo la geografía y las ciencias políticas, han propuesto marcos teóricos que, sin ignorar el papel del Estado, abren vertientes de análisis político-territoriales tales como la política-práctica-percepción (PPP), además de las realistas, funcionalistas y neo-funcionalistas, y la gobernanza multinivel dentro de la disciplina de las relaciones internacionales (Kolossov, 2005; Brunet-Jailly, 2006; Kolossov y Scott, 2013). Tanto el ppp y el enfoque de la gobernanza multinivel incorporan el estudio del Estado y de las nuevas configuraciones políticas utilizando escalas y niveles para dar cuenta del diseño y puesta en práctica de políticas (local, subregional, nacional, regional, entre otras) (Koff, 2008). Muchos estudios tienden a centrarse en las percepciones sociológicas, antropológicas y geográficas acerca de la vida, las venturas y desventuras de la población fronteriza (Martínez, 1997; Paasi, 1999; Kearney, 1998; Observatorio de Venezuela, 2018; Observatorio de Venezuela, 2019; Mouly, Idler y Garrido, 2015; Bressan, 2016).

La literatura sobre seguridad tiende a definirla respecto a las amenazas y miedos, y si se está más o menos seguro según la amenaza percibida o el miedo percibido y el poder para enfrentarlas (Orozco, 2006). Tradicionalmente la seguridad en el campo de las relaciones internacionales se ha centrado en poder y conflicto y han sido enfocados, inicialmente, desde el realismo, idealismo y groscianismo. Cada uno ha dado origen a modelos de seguridad y ha permitido en su conjunto acuñar conceptos como el de seguridad social, colectiva, democrática, global e internacional, entre otras. Ahora bien, lo público ha dado origen a la seguridad del Estado y esta se subdivide en: la propia del 
Estado (militar) y la del ciudadano (seguridad pública) (Orozco, 2006; Arteaga, 2012).

Los estudios y análisis sobre seguridad previos a 11/09 estaban pasando a ser eje articulador de las relaciones internacionales (Orozco, 2006) en la cual se dio inicio a la etapa interméstica mediante la cual la seguridad del Estado se refiere a su capacidad para enfrentar amenazas y desafíos internos y externos que venía caracterizando las doctrinas de seguridad y defensa de la nación (González y Betancourt, 2018). La seguridad también se interpretó como resultado de la identidad de los grupos, colectividades e instituciones del sistema que construyen lazos de solidaridad infranqueables. Siguiendo a Orozco, otro giro importante de la seguridad fue la preservación del ser humano y de "la garantía de las condiciones materiales necesarias para el desarrollo libre de sus capacidades" (p. 167) lo que llevó al surgimiento de conceptos y modelos como el de seguridad global, que ponía el acento en la responsabilidad de la comunidad internacional para proteger la necesidad de los pueblos, dejando de lado o acompañando al Estado, según el caso, en el diseño de las políticas de seguridad para proteger a la población vulnerable.

En Latinoamérica, la seguridad deja de ser vista únicamente como seguridad y defensa del Estado y su territorio (especialmente en sus confines) para incorporar al ciudadano a la seguridad pública y a la administración de justicia, aunque la teoría y la práctica de la misma continúen permeadas por la seguridad, la defensa, la territorialidad y la soberanía (Arteaga, 2012; Peña, Sierra-Zamora y Hoyos, 2019). La estrecha e histórica relación con Estados Unidos es un elemento a destacar en la definición y puesta en práctica de estrategias y políticas de seguridad internacional en Latinoamérica, en particular en Colombia donde se destacan percepciones similares del enemigo y las amenazas entre las que se incluye el comunismo y la lucha contra las drogas; al igual que respuestas y alianzas multilaterales de acción (Rodríguez, 2015; González y Betancourt, 2018; Hernández y Romero-Arias, 2020).

Las políticas de seguridad y fronteras aceptaban la idea de que el Estado-nación se diluiría más rápido, que los Estados Unidos era el único súper poder militar y que vendrían tiempos de paz duradera (Human Security Centre, 2005), en donde los conflictos serían más domésticos que internacionales (Kolossov, 2005; Kolossov y Scott, 2013). Los conceptos y las políticas de seguridad que se adaptaban al enfoque realista daban paso a enfoques alternativos relacionadas con la "seguridad humana", la cual "en vez de centrarse en los intereses del Estado-Nación, se concentraban en los intereses de los seres humanos y en cómo protegerlos y proteger sus comunidades de peligros" (Farson, 2006, p. 29). El enfoque de la interdependencia compleja de Keohane y Nye (1987) se aceptaba para explicar las dinámicas fronterizas y ello implicaba el reconocimiento de las complejas interacciones subyacentes entre los Estados y el soslayo de las acciones militares para defenderse o protegerse del otro.

El 11/09 produjo una reacción drástica e inmediata y el retorno a la visión realista. Una vez más, los intereses nacionales y el Estadonación ocuparon prioridad en la agenda de la política exterior, de seguridad y fronteriza. El impacto más inmediato y visible tomó lugar en la percepción de la seguridad y en las fronteras. 
También dio fuerza a los ejecutivos nacionales como decisores y diseñadores de la política de seguridad nacional y fronteriza en lo particular (Hernández y Romero-Arias, 2020). Las adaptaciones para incorporar otros niveles y actores sociales en su diseño se reducen, en los países donde habían sido incorporadas, o no se producen, como en el caso de Venezuela.

Las fronteras se convierten en una amenaza a la seguridad de Estados Unidos (BrunetJailly, 2006; Rodríguez, 2015; Hernández y Romero-Arias, 2020) en primer lugar y del resto del mundo paulatinamente en donde factores migratorios se unen a la amenaza global del terrorismo. El comercio, inversión, migración abierta y lucha contra el narcotráfico pasaron a ocupar un segundo lugar después de la "guerra al terrorismo" y la seguridad nacional global y en las fronteras (Ramos y Woo, 2004; Bailey y Chabat, 2002).

Los estudios de seguridad, tradicionalmente otorgan al Estado (y generalmente al ejecutivo) la función de garantes y en un mayor o menor grado la seguridad depende de la eficiencia del Estado. Y a pesar que el cambio hacia estudios que enfoquen la seguridad pública (ciudadana) se mantiene como área de estudio, no hay preocupación particular por examinar la seguridad pública en fronteras.

Quienes estudian la seguridad pública en Latinoamérica la ven como consecuencia de factores sociales generadores (pobreza y desigualdad), conflicto entre grupos sociales (lucha de clases: victimario-víctima), formas simbólicas de identidad cultural (estatus) y fragmentación urbana entre otros (Arteaga, 2012). Poco o nada se dedica al estudio de la seguridad pública en contextos de amenazas o temores (aparentes o reales) derivados de la debilidad y la fragmentación estatal y del predominio de prácticas de seguridad y defensa (militar) como es el caso de la seguridad en las fronteras y en la frontera colombo-venezolana, donde la "guerra contra el terrorismo", surgida luego del 11/09 de 2001 en Estados Unidos, y a la cual se incorpora a los grupos armados/ insurgentes ilegales como parte de la política de su seguridad internacional, y que Colombia respalda y asume mediante la PSD que combina la lucha antinarcóticos y la contrainsurgente (González y Betancourt, 2018). En esta frontera, la falta de legitimidad del Estado, derivada del escaso control sobre el territorio y la población, se combate con militarización, como el recurso más efectivo y probado en materia de seguridad pública del Estado, para centralizar y recuperar el control territorial en zonas capturadas por el crimen organizado (Hernández y Romero-Arias, 2020). Es decir, es la estrategia que permite fortalecer al ejecutivo en situaciones convulsas y amenazas intermésticas. El desencuentro con la política de seguridad venezolana, que aunque igualmente opta por la militarización para garantizar la legitimidad del gobierno persigue objetivos opuestos a Colombia. En ambas situaciones los conceptos y prácticas de seguridad ignoran al ciudadano y refuerzan lo militar.

El estudio de la seguridad fronteriza en Colombia-Venezuela y en Suramérica ha sido escaso, a pesar de su relevancia para el futuro de la zona de frontera. La mayor parte de la literatura acerca de las relaciones entre Colombia y Venezuela en los últimos años se ha centrado en las diferencias ideológicas entre los dos gobiernos (Páez, 2009; Cardoso, 2010 y 2011; 
International Crisis Group, 2004 y 2011) en la alineación del gobierno colombiano con la política de seguridad de los Estados Unidos luego del 11 de septiembre de 2001 (Andrade, 2005). Pero el efecto de la PSD en la frontera común no se ha estudiado.

Algunos de los trabajos más recientes sobre seguridad en las fronteras incluyen el de Fernando Carrión y Johanna Espin (2011), quienes se han dedicado a investigar la gobernanza de la seguridad ciudadana, enfatizando en la necesidad de adelantar políticas domésticas e internacionales comunes para evitar y disminuir el flagelo de la inseguridad y los impactos sociales, económicos, políticos e incluso ambientales. Por su parte, Jenne (2014) revisa la agenda de seguridad fronteriza de Colombia y los efectos transversales en el caso colombovenezolano, destacando que las zonas fronterizas aún adolecen de presencia significativa del Estado y son, además, el escenario donde se concentran amenazas como el narcotráfico, el crimen organizado y los grupos armados no estatales. También destaca que los problemas limítrofes aún pendientes siguen permeando la agenda de seguridad en detrimento de la atención a las nuevas amenazas. Morffe (2014) analiza el riesgo percibido en la seguridad ciudadana desde el habitante de frontera, mediante un estudio cualitativo, para concluir que la frontera común tiene problemas de seguridad, incluyendo violencia, secuestros, "extorsión", narcotráfico, entre otras actividades criminales. La coordinación internacional entre los dos países ha sido problemática.

La revisión nos muestra que no hay estudios sobre la PSD y su percepción en la frontera colombo-venezolana o en la de TNs, lo que permite destacar la importancia del estudio dado que la misma fue eje central del gobierno de Álvaro Uribe y fuente de conflicto entre Colombia y Venezuela por lo que su impacto en la frontera tiene valor para comprender los cambios en las dinámicas fronterizas y si la PSD ha sido fuente de inestabilidad e inseguridad, con sus consecuentes efectos en la seguridad humana y el desarrollo regional fronterizo.

\section{METODOLOGÍA}

El estudio es de campo, exploratorio y cualitativo en el que se hace una aproximación a los diferentes constructos y categorías implicadas. Una de las características fundamentales de la investigación cualitativa es la importancia que se atribuye al contexto. La investigación de campo se desarrolló y obtuvo datos directamente en San Cristóbal y San Antonio-Ureña por el lado de Venezuela y San José de Cúcuta, Villa del Rosario y La Parada por el lado colombiano, por lo que las poblaciones están ubicadas en el espacio fronterizo entre Táchira y Norte de Santander y se convierten en ciudades de tránsito transfronterizo.

Se adopta la teoría fundamentada (Glasser y Strauss, 1967; Strauss y Corbin, 2002) para obtener teoría desde los datos. Resaltan en esta teoría el muestreo teórico, la comparación constante y la codificación teórica. En el muestreo teórico el autor decide los sujetos $\mathrm{u}$ objetos que le proporcionan información relevante (Flick, 2004). La decisión sobre el volumen de los datos es la saturación teórica, entendida como "un criterio para juzgar cuándo dejar de muestrear los diferentes grupos relevantes a una categoría" (Glaser \& Strauss, 
1967, p. 61). A continuación se describe el proceso seguido.

Las entrevistas se caracterizaron por ser a profundidad ya que se realizaron en varias etapas. Las preguntas fueron abiertas y el orden se adecuó a los participantes; se creó la confianza por carácter amistoso; atendieron el contexto social y se ajustaron al lenguaje del entrevistado. Se conversó, en repetidas oportunidades, con los informantes, pues era necesario un clima psicológico de cordialidad y confianza. Una vez logrado se realizó la entrevista formal a cada informante en la que se constató un intercambio comunicacional fluido y permanente. En total fueron seis preguntas, a saber:

1. Cambios principales que trajeron a la frontera las iniciativas de la PSD y PCSD de Colombia.

2. Influencia de los desencuentros gubernamentales en la dinámica fronteriza.

3. Militarización de la comunidad nortesantandereana/tachirense con la PSD.

4. Grupos de la comunidad (en Norte de Santander y Táchira) más afectados por los cambios en la política de seguridad de Colombia.

5. Adaptación de los distintos sectores de la comunidad a las realidades cambiantes en la frontera y en los cruces fronterizos.

6. Percepción sobre la región fronteriza de Táchira-Norte de Santander y cómo ha continuado cambiando después de esta fecha.

Las entrevistas se realizaron durante tres años y medio (enero 2015-julio 2019) debido a las dificultades logísticas y de agenda de los entrevistados, así como a los cambios de gobiernos regionales y locales.
Se partió de una estimación de diez informantes, que se fue elevando, en parte, con la utilización del muestreo en cadena (Hernández, Fernández y Baptista, 2014), y en parte, por la negativa de informantes a aportar información que consideraron sensitiva y que les generaba temores de correr riesgos importantes. El número de informantes contactados se elevó a 50, y a partir de allí se eliminó a quienes reusaban profundizar y aportar información relevante o de calidad. Ello contribuyó a que el tiempo estimado para realizar el trabajo se extendiera significativamente. Se buscó entrevistar a las autoridades nacionales, regionales y locales de cada país; y a los grupos sociales más relevantes de la frontera (comerciantes, industriales y profesionales) con el fin de obtener la información que permitiera lograr los objetivos.

En definitiva, se hizo una aproximación a 50 actores ( 25 de cada lado) y se obtuvieron 15 respuestas, de las cuales solo 8 aportan información relevante por lo que se optó por trabajar con las respuestas de esos 8 informantes. La distribución final de los informantes es la siguiente: ver tablas 1 y 2 .

El estudio de las percepciones implica adentrarse en el mundo individual y subjetivo del informante. Es conocer la forma en que los factores externos (culturales y sociales) influyen en la percepción que el individuo tiene de sí mismo y de su entorno. Se refiere a procesos individuales vinculados a "mecanismos sensoriales y de cognición” (Atar, 2010, p. 10) y, en consecuencia, significa analizar cómo los individuos o los grupos se sitúan ante determinados objetos.

La información obtenida se analizó conforme al procedimiento sugerido por Hernán- 
Tabla 1

Distribución de informantes que aportan información relevante

\begin{tabular}{|l|c|c|}
\hline \multicolumn{1}{|c|}{ INFORMANTE } & COLOMBIA & VENEZUELA \\
\hline De política nacional (gobierno, economía) & 2 & - \\
\hline De política regional/local (gobierno economía) & 2 & 1 \\
\hline Actores locales & 1 & 2 \\
\hline TOTAL & 5 & 3 \\
\hline
\end{tabular}

Tabla 2

Caracterización de los informantes

\begin{tabular}{|c|l|l|l|}
\hline NÚMERO & IDENTIFICACIÓN & \multicolumn{1}{|c|}{ SECTOR } & \multicolumn{1}{|c|}{ ESCENARIO } \\
\hline 1 & S1 & Gubernamental & Colombia, nacional \\
\hline 2 & S2 & Gubernamental & Colombia, nacional \\
\hline 3 & S3 & Gubernamental & Colombia, regional \\
\hline 4 & S4 & Gubernamental & Colombia, local \\
\hline 5 & S5 & Gubernamental & Venezuela, regional \\
\hline 6 & S6 & Privado & Frontera \\
\hline 7 & S7 & Privado & Frontera \\
\hline 8 & S8 & Privado & Frontera \\
\hline
\end{tabular}

dez, Fernández y Baptista (2014): (a) organización de los datos para verificar su pertinencia y calidad; (b) transcripción del material; (c) análisis, para lo cual se desarrolló un proceso de codificación abierta que permitió generar las siguientes categorías:

a. Ambivalencia sobre la capacidad del Estado.

b. Oscilaciones en la credibilidad del Estado.

c. Pérdida de la calidad de vida.

Este procedimiento se realizó de forma manual y con el mismo se estima existe el rigor científico relacionado con la ética de la investigación y del investigador (Sandin, 2013). Se incluyen: privacidad y confidencialidad; consentimiento informado y estancia en el campo. La privacidad y confidencialidad condujeron a la protección de la identidad de los informantes y se evitó suministrar información que permitiera su identificación. De la misma manera, se explicó a los entrevistados el propósito de la investigación, se solicitó su consentimiento para el uso de su información $y$, finalmente, se aseguró una estancia adecuada en el campo; la cual comenzó con los primeros contactos en cada uno de las poblaciones de la frontera estudiada, lectura de la prensa escrita y digital, y se extendió por el tiempo necesario hasta alcanzar la necesaria confianza para la realización de la entrevista. Producto del trabajo desarrollado se obtuvieron los resultados que se presentan a continuación. 


\section{RESULTADOS}

En adelante se presentan los resultados de las entrevistas según las categorías surgidas del trabajo de campo y las interrogantes planteadas en las entrevistas: Ambivalencia sobre la capacidad del Estado, oscilaciones en la credibilidad del Estado y pérdida de la calidad de vida. Dichas categorías se subdividen en dimensiones.

\subsection{Ambivalencia sobre la capacidad del Estado}

Los informantes perciben distintas situaciones vinculadas al papel, fortalecimiento, militarización y debilidad del Estado entre la que se incluye la falta de alcanzar acuerdos con Venezuela, otros que lucen contradictorios y que permiten denominar a esta categoría: ambivalencia sobre la capacidad del Estado. Así, surgen dimensiones tales como: fortalecimiento, debilidad del Estado y aumento de la violencia.

4.1.a. Fortalecimiento del Estado: Es considerado como el esfuerzo del Estado por establecer el control del territorio, y de la población como legítimo garante de la violencia, la militarización y recuperación de la institucionalidad. Es expresado como positivo y con opiniones como la de "...la percepción de un Estado Fuerte” (S5) y la “...recuperación del control del Estado sobre la mayor parte del territorio nacional..." (S5), el "...ganarle terreno a estos grupos alzados en armas en las fronteras, pues el aparato militar colombiano empieza a tener credibilidad y empieza a adelantar sus fichas, a mover sus ejércitos (S7).
"La PSD es una política nacional que se centró en el copamiento y en la necesidad estricta que tiene Colombia, que los actores del Estado colombiano tengan el monopolio de la fuerza y el monopolio del orden y de la restauración de la legalidad" (S7) y así, para algunos se evidenció “...por ejemplo en el Municipio del Tarra, se creó la Brigada Móvil No. 23 del Ejército Nacional, y se aumentó el pie de fuerza de la policía acantonada en la estación de policía se pasó de 12 miembros a $25 . . . ”(S 1)$, en el Catatumbo nortesantandereano y en otras zonas con la puesta en práctica de "nuevos esquemas y divisiones militares" (S2) que en detalle evidencian la militarización y confianza de la población en la misma:

Aumentó la militarización el pie de fuerza... Y se empezó a ver más pie de fuerza en las fronteras. Empezaron las fuerzas de tarea Omega, las fuerzas de tarea Vulcano. Se vio como toda la ayuda militar de los Estados Unidos empezó a llegar a Colombia... se notó de manera importante el aumento del pie de fuerza, armamentos más sofisticados, aumento de la credibilidad de los militares. El gobierno les dio suficiencia para poder funcionar, los estaba amparando y pues obviamente se estaba viendo el trabajo que se estaba haciendo (S7).

También

...bajo un principio de restablecimiento de la fuerza y seguridad de la autoridad pública,... devolver el espacio a muchos alcaldes, que si bien nos acordamos, eran más de 300, casi 400 alcaldes que no habían podido ejercer desde su territorio. Y primero el restablecimiento de esa autoridad, tanto la local, como la regional, como la nacional era necesario y 
por eso se desplazó todo ese componente fuerte, de fortalecimiento del ejército, de fortalecimiento de la autoridad pero combinado también con un ejercicio de desarrollo comunitario importante y haciendo de Colombia un Estado comunitario donde sabemos que las mismas bases deben producir el ejercicio de las propuestas de los proyectos de desarrollo local (S2).

En fin, aumentó la “...institucionalidad y la legalidad... cuando los alcaldes han podido volver a sus municipios" (S2).

4.1.b. Debilidad del Estado: Se asume como tal a la asociación del componente armado del Estado con los grupos alzados en armas contra el Estado y las limitaciones del gobierno para lograr acuerdos o reconocimiento de la PSD por parte de Venezuela. Así el avance en fortalecimiento Estatal se vio empañado por la opacidad y la asociación del Estado con las Autodefensas Unidas de Colombia.

La opinión generalizada es que "La seguridad brindada era compartida entre la policía, el ejército y lo grupos de autodefensas, más conocidas como AUC...." (S4). "La SD inicialmente empieza a funcionar con el apoyo del Estado a los grupos paramilitares" (S7). En definitiva, se recurrió a métodos poco ortodoxos.

... la presencia de las AUC, con comandancia de Mancuso, el Iguano, entre otros, llegó a hacer operaciones paramilitares con acciones violentas de muerte a productores, recolectores, transportadores y otros vinculados a estas actividades del narcotráfico. Las AUc coparon casi todo el NS donde hicieron "limpieza" contrainsurgente con asesinatos, desapariciones y migración forzosa en gran número. Hubo también bastantes homicidios de personas inocentes... (S6)
Adicionalmente se destaca que la PSD evidenció el incremento de la falta de cooperación por parte de Venezuela, lo que deterioró la institucionalidad estatal colombiana en el escenario suramericano. Es decir, el poder internacional del Estado se afectó negativamente con la PSD y con los desencuentros con Venezuela. Se “... exigía se reforzara la seguridad de las fronteras a fin de neutralizar ... bandas criminales, Bacrim, ... hizo falta ... colaboración de las fuerzas armadas de Venezuela que no ... en frenar a los criminales que huían desde Colombia..." (S1) y “... en las fronteras... los grupos al margen de la ley” se fueron “... más allá de la línea de frontera para resguardarse y mantener una posibilidad de acción desde detrás de la línea fronteriza; lo que le permitía establecer como una especie de burladero a la fuerza armada" (S2) “... de no haber ocurrido tantos desencuentros la lucha contra las Bacrim había sido más eficiente pues... muchos de los delincuentes huían hacía Venezuela” (S1).

También hubo pérdidas en la cooperación e integración binacional que afectaron la frontera "Los dos países perdieron demasiado, tanto en lo político, lo económico y lo social”. “...Con la salida de Venezuela de la CAN, el dinamismo económico binacional se vio afectado... la ZIF..." (S6) fue uno de ellos y, "Los históricos lazos de cooperación entre las poblaciones de Norte de Santander y Táchira, entre sus gobernantes, empresarios y sociedad civil se fueron deteriorando lentamente" (S8). "Se fueron perdiendo los espacios de un diálogo constructivo y de un actuar concertado entre nortesantandereanos y tachirenses. Las reuniones de gobernadores y alcaldes fronterizos y gremios se fueron haciendo cada vez 
menos frecuentes y con intereses cada vez más distantes (S8).

4.1.c. Aumento de la violencia: Aunque forma parte de la debilidad del Estado, merece un segmento aparte dado el énfasis en el control y recuperación del territorio mediante incursiones militares. La violencia legítima del Estado se destacó en las dimensiones fortalecimiento y debilidad del Estado, donde si bien es apreciada también es criticada por su relacionamiento con grupos alzados en armas al margen de la ley (entiéndase las AUC, entre otros, con la excepción de las Farc). Y es la violencia de estos últimos, en asociación con sectores de las Fuerzas Armadas Nacionales, a los que se le atribuyen mayores actos de violencia y el predominio de la opacidad y secretismo propios de las actividades militares que dificultan una evaluación aproximada y transparente de la PSD.

En primer lugar se destaca que "los paramilitares tenían todo el poder de las fronteras durante la SD”, e imponían el control mediante la violencia ilegítima. "Existieron esos hornos donde metían a la gente, donde cremaban a la gente, quien no estuviese de acuerdo... las leyes que los paramilitares colocaban en la frontera,..., la gente era cremada y desaparecida en esos hornos aquí en la frontera del Norte de Santander” (S7). Es decir, “...es la población civil, las personas que no están metidas en el conflicto... pierden sus hijos, pierden sus padres, desplazamientos forzados, desapariciones, secuestros... Entonces esta población de frontera, que no estaba inmiscuida en el conflicto, es la que se ve más afectada..." (S7) por la violencia.

Así, hay quienes señalan que hubo “... sectores menos favorecidos, pero con menos peso en la opinión pública,... Especialmente la población desplazada la gran mayoría por el accionar de los paramilitares" (S8), que hubo abusos que afectaron a los sectores rurales “...una forma generalizada en los campesinos (áreas rurales). Familias enteras fueron masacradas y sus propiedades destruidas por incendios premeditados,...en muchos casos, tener que vender predios en el valor que les pagaran los paramilitares y desplazarse para no ser asesinados" (S4). "Los grupos de población desplazada que eran campesinos de las zonas del Catatumbo y aledaños a estos territorios indígenas y personas dedicadas a las actividades de la coca" (S6), que fueron objeto de la violencia de "las Auc, del ejército y la policía" (S7).

En segundo lugar crecen "... estructuras más ilegales y más fuertes en las zonas de frontera" (S2), y se produce un aumento de los delitos “...con los grupos al margen de la ley se incrementaban los delitos de impacto (extorsión, homicidio, secuestro en zonas de frontera) (S3) por lo que, se "produjo un impacto negativo" (S7).

En resumen, las percepciones sobre la incidencia de la PSD muestran que la capacidad y poder del Estado sobre el territorio, aunque deficiente, avanzó significativamente a pesar que no alcanzó a producir una recuperación institucional que permitiera la preeminencia del Estado (gobierno) como bien superior a recuperar y construir en Colombia y en su relacionamiento internacional.

\subsection{Oscilaciones en la credibilidad del Estado}

La percepción generalizada es que la credibilidad del Estado tuvo dos momentos claramente 
diferenciados: El primero de confianza y credibilidad general y creciente en la capacidad y uso de la violencia legítima, y el segundo de críticas y desconfianza que se destaca en las dudas sobre la puesta en práctica de la PSD.

En Norte de Santander hubo períodos de relativa calma: "el principal cambio que se tuvo a lo largo de la frontera T-NS fue volver a sentirnos seguros y libres de la extorsión a que éramos sometidos por la guerrilla del EPL y ELN" (S4), el departamento creció y estuvo "apto para todo tipo de actividades comerciales, industriales y agrícolas" (S4). También se destaca la protección brindada a la sociedad en general porque "ningún grupo social se afectó negativamente, contrariamente los gremios sociales y defensores de DD.HH. eran protegidos por el Estado" (S3) a defensores de derechos humanos "... se les brindaba protección... la sensación de seguridad en los habitantes del lado colombiano empezó a crecer... La SD del expresidente Uribe permitió que las civiles se incorporaran a este proceso colaborando con información y trabajando en equipo con las autoridades" (S1). En definitiva, "Los comerciantes y grupos de la sociedad civil de ambos países la aceptan porque buscan mejorar las relaciones y las condiciones de vida de sus ciudadanos" (S5). En síntesis la percepción es que

la gran mayoría de sectores sociales en Norte de Santander se sintieron beneficiados ...el poder movilizarse por las carreteras, la sensación de la derrota a las guerrillas, la llegada de batallones antiguerrillas, la disminución de los secuestros y las extorsiones le dieron a la población una fuerte sensación de que se estaba recuperando la seguridad y el orden social (S8).
La credibilidad y confianza se deteriora al percatarse de las consecuencias negativas para la población. Así, los efectos “....son diversos, ...lo que ha propiciado es una pérdida de confianza" y se termina "... haciendo una fractura en la interlocución necesaria de... los ejércitos, las policías... las autoridades que luchan contra el contrabando y la ilegalidad..." (S2).

Para la población, la "...presencia militar...” (S4) estuvo acompañada de violaciones a distintos derechos humanos y que ya empezaban a hacerse públicos para 2010. Entonces, "al 2010 ya se hablaba de los abusos de las AUC en todos los órdenes: en lo social se abusaba de niñas y jóvenes al ser convertidas en esclavas sexuales además de la práctica de abortos en los campamentos, las masacres de campesinos, los falsos positivos..." (S4) y desde el punto de vista económico se observaba "la extorsión a empresarios y los asesinatos a quienes no 'colaboraban' (S4). Pero es solo una década más tarde cuando se estima están apareciendo casos que evidencian los desmanes y violencia, y que en definitiva restan credibilidad a la psd.

\subsection{Pérdida de la calidad de vida}

La calidad de vida tiende a asociarse a necesidades básicas satisfechas, libertades y seguridad pública que, acompañadas de políticas del Estado adecuadas, permitan el desarrollo y la potenciación de la capacidad creadora y de cualidades de creatividad y adaptabilidad para hacer un mejor uso de los recursos. La pérdida o deterioro del acceso a la satisfacción de necesidades y de las libertades básicas, así como de la seguridad pública, aunado a la falta de políticas de desarrollo apropiadas, aun 
cuando no disminuyan la capacidad creadora y la adaptabilidad de la población hacen que dichos esfuerzos y resultados sean menos halagadores. De esta aproximación al tema surgen las siguientes dimensiones:

4.3.a. Aumento de desempleo: El caso que el comercio fronterizo se mantuviera sin grandes cambios no explica el deterioro de las condiciones de vida de la población. San José de Cúcuta se muestra como "la primera ciudad en índice de desempleo" (S4) de Colombia. Se destacan variaciones por períodos ya que "Se pasó de la prosperidad y seguridad (20022007), a "la incertidumbre, desplazamiento, retorno a la violencia y a la criminalidad de 2008 en adelante..." (S4), en muchas zonas de Norte de Santander, en especial hacia Sardinata y Catatumbo en general disminuyeron el empleo y subempleo.

4.3.b. Éxodo poblacional: El aumento de la violencia contribuyó decididamente a que, en general, la población civil optara por desplazarse o irse a otras zonas de los países. Para 2016, el informante S2 destaca que los indicadores sociodemográficos de Colombia muestran que

... Colombia está perdiendo el 1\% de la población en zonas de frontera. Hace 26 años, el 25\% de la población colombiana estaba ubicada en zonas limítrofes y de frontera... Hoy día, de ese $25 \%$ estamos en el $9 \%$... La salida de la población de las zonas de frontera es un indicador tremendamente preocupante y es donde yo creo que tenemos que hacer énfasis para que la frontera tenga una buena perspectiva (S2).

Según este informante, ese año confirma estadísticamente la tendencia de pérdida de población de la década anterior.

\section{3.c. Pérdida de libertades y de la seguridad} pública: Las restricciones y limitaciones a la libertad de información y expresión se destacan con la opinión de que “... no teníamos acceso total a muchas cosas... Había cosas que no podíamos registrar, decir. Teníamos que hacer silencio... porque el gobierno... no tenía control con lo que ocurría con los grupos alzados en armas ahí en la frontera..." (S7).

En cuanto a la seguridad pública se argumenta que la intención del gobierno de “... fortalecer las actividades y presencia de los órganos de seguridad a lo largo de las fronteras y en todo el territorio nacional..." (S3) tuvo sus oscilaciones. Hay quienes consideran que hubo pacificación inicialmente y que la inseguridad retornó luego porque "En materia de seguridad en el año 2010, que recuerde, no había tanta presencia de insurgencia guerrillera en las zonas fronterizas..." (S1), pero "Muchas de las democracias locales fueron permeadas por dineros del contrabando de la gasolina, del narcotráfico, del mismo conflicto y las últimas dos décadas nos han permitido un poco ponerle el reflector a la frontera..." (S2).

Norte de Santander

...es uno de los más afectados por la violencia del conflicto colombiano en las últimas tres décadas. En particular el Catatumbo ha sido una de las regiones más disputadas por todos los grupos al margen de la ley por los cultivos de coca y su cercanía a Venezuela (S8).

Y a pesar que en la categoría oscilaciones en la credibilidad del Estado se informa que hubo respeto por las organizaciones de derechos hu- 
manos, las dimensiones debilidad del Estado y aumento de la violencia mostraron que hubo severas violaciones de DD.HH.; por lo que las libertades básicas y la seguridad pública estuvieron muy amenazadas.

4.3.d. Falta de políticas de acompañamiento: Para la población "es difícil adaptarse,... hacen que todo sea impredecible... Nuestras instituciones se ven obligadas a adaptarse a estos vaivenes para tratar de apoyar y controlar lo que de allí surge" (S1). Pero predomina en "lo económico", se fortalece "el rebusque" diario (S4) sin mayores apoyos en políticas sociales o económicas.

A lo anterior se contrapone la percepción de que en ningún momento el orden "...no lo tenían los Estados, sobre todo el Estado colombiano y creo que el venezolano tampoco,... durante ese tiempo nunca han tenido control de sus fronteras" (S7). Pero... la percepción de la gente "durante todo este tiempo fue, del predominio del desgobierno, cero desarrollo, cero propuestas para la gente de la frontera, perdido el orden de la frontera,..." (S7). Así, para algunos "... la frontera es una parte de la sociedad muy noble con exigencias particulares que no son atendidas como prioritarias por ningún gobierno..." (S5).

4.3.e. Resiliencia: En términos generales, los distintos actores destacan que la población fronteriza tiene "la ventaja ... que está en constantes cambios, son dinámicas, se adaptan muy rápidamente a las nuevas condiciones" (S5) y que "en general las comunidades fronterizas se van adaptando a las condiciones de los actores dominantes en cada momento..., tratando de mantener su modo de vivir a partir de la relación fronteriza” (S8), “... actualmente esta masa llamada frontera sigue mostrando su lado más amable y dinámico siendo en la práctica un canal humanitario de hecho y no por decreto" (S4).

4.3.f. Creatividad: La creatividad para afrontar las amenazas y cambios, según los entrevistados, mantuvo el comercio binacional fronterizo sin grandes cambios, con la excepción de las fluctuaciones derivadas del “... cierre de los pasos... el diario cruce de la frontera y las diversas actividades que de ello se desprenden no cambiaron de forma radical" (S5).

Finalmente, la revisión de las percepciones a las interrogantes planteadas coinciden en afirmar que la frontera de TNs fue una zona muy afectada por la PSD debido a que, territorialmente, su énfasis fue el centro del país y la recuperación de la movilidad. En la frontera el gobierno optó por asociarse con otros grupos al margen de la ley para combatir a las Farc. En consecuencia, la frontera, sobre todo la colombo-venezolana en TNS, si bien fue altamente militarizada mostró más inseguridad pública y poca recuperación del poder del Estado debido a las alianzas que realizó con grupos ilegales a fin de combatir la guerrilla.

\section{CONCLUSIONES}

Esta investigación dio respuesta a las interrogantes planteadas y mostró que los cambios en la PSD de Colombia tuvo efectos varios. Contribuyó a fortalecer la capacidad del Estado, sin llegar a considerarse suficiente, al mismo tiempo que fue percibida con impactos negativos en la dinámica del TNs y en la seguridad pública fronteriza. 
Ahora bien, a pesar de las distintas formas de percibir la PSD, las categorías emergentes de los informantes: Ambivalencia sobre la capacidad del Estado, oscilaciones en la credibilidad del Estado y pérdida de la calidad de vida y sus dimensiones (fortalecimiento y debilidad del Estado, violencia, éxodo poblacional, desempleo, falta de acompañamiento con políticas de desarrollo, pérdida de libertades y seguridad pública y resiliencia, entre otras) nos muestran que la PSD abonó en la recuperación estatal, pero muestran igualmente un Estado fragmentado y con grandes limitaciones para aumentar su capacidad. Las explicaciones avanzadas van desde la construcción misma del Estado colombiano, la historia de violencia y conflicto armado así como la incapacidad del gobierno por cohesionar y dar cierta homogeneidad a la nación. Y sobre esto se ha investigado ampliamente (Granada, Restrepo y Vargas 2009; Elhawary, 2008; Gutiérrez, Wills y Sánchez, 2005; Orjuela, 2000).

Ahora bien, las percepciones encontradas sobre la seguridad democrática en la frontera Táchira-Norte de Santander, en el período referido, no parecen haberse modificado sustancialmente según lo muestra Jenne (2014) al destacar la persistencia de amenazas e inseguridad cuatro años después. El proceso de paz iniciado con la firma del Acuerdo del Teatro Colón entre la Farc-EP y el gobierno nacional en 2016 y las oscilaciones del pos-acuerdo poco han ayudado a reducir la conflictividad actual de este territorio en donde lo ilegal autóctono se conjuga con lo transnacional y crean una dinámica delictiva que amenaza la seguridad de Colombia, Venezuela y la región.
Las categorías emergentes, obtenidas mediante las interrogantes planteadas, como elementos comunes tiende igualmente a resaltar las ambivalencias y limitaciones del Estado para crear credibilidad, y esto se convierte en negativo al evaluar las opciones de seguridad pública para el ciudadano y la ciudadanía en la frontera de TNS; en donde la policía, órganos de seguimiento y control de las políticas públicas dejaron de cumplir el propósito principal de proteger al ciudadano, crear confianza en el gobierno para evitar el retorno a las armas o a la continuidad de actos delictivos; en fin brindar una seguridad más humana. Puede afirmarse, entonces, que la guerra al terrorismo sin los correspondientes balances y controles permitió excesos y violencia. Pero también, y muy probablemente, era necesaria una acción militar de debilitamiento de los grupos alzados en armas para recuperar el poder y control del Estado que se logró. Es decir, la PSD, a pesar de sus logros, mostró errores importantes y en la frontera se observaron y vivieron profundamente y por ello los entrevistados mostraron esas ambivalencias. Obviamente hay factores externos como la desarticulación con Venezuela que no pueden atribuírsele totalmente.

Para uno de los entrevistados, los 18 años de "la línea de la seguridad militar" (S2) han dado como resultado evaluaciones y estudios especializados que dan cuenta de "...indicadores de derechos humanos... desarrollo económico y el crecimiento de las economías legales,... narcotráfico y áreas sembradas de cultivos ilícitos... la institucionalidad y la legalidad... cuando los alcaldes han podido volver a sus municipios" (S2) en Colombia y se ha hecho alguna aproximación a la fronte- 
ra. Sin embargo, esas líneas de investigación no se han debatido y cruzado para realizar una evaluación desapasionada de la PSD que si bien luce como una política implementada y pasada, sigue formando parte de las políticas del gobierno nacional en cuanto a la oferta y práctica de una "seguridad militar", en especial en la frontera donde se ha combinado "con los fenómenos históricos de ilegalidad que existían como el contrabando, el narcotráfico, por decir unos cuantos" (S2).

Una evaluación académica de la PSD en la frontera pasa por buscar respuestas y explorar medios para reducir la tendencia al despoblamiento, aumentar la legalidad y la cooperación vecinal con Venezuela.

En definitiva, es importante acompañar y explorar la política de seguridad militar con la pública y ciudadana para producir conocimiento y orientar a los diseñadores de las políticas de seguridad en caminos a seguir en la construcción de institucionalidad, de recuperación del Estado de derecho y de capacidad estatal, que permitan a Colombia, pero también a Latinoamérica, superar la pesada carga de estructuras estatales surgidas en la o con la dictadura y de Estados militarizados.

Adicionalmente, por lo impactante, la PSD reclama mayores investigaciones y más transparencia para avanzar en la reconstrucción institucional del Estado. Al respecto, y en general "....cualquier política nacional tiene... primero un buen propósito y segundo tiene efectos positivos, efectos poco visibles y efectos negativos... una valoración de esa política todavía requiere un mayor grado de información..." (S2)

\section{REFERENCIAS}

Andrade, P. (editor) y Olano, A. (2005). Constitucionalismo autoritario: los regímenes contemporáneos en la Región Andina, Ecuador: Universidad Andina Simón Bolívar.

Arteaga Botello, N. (2012). Surveillance Studies: An Agenda for Latin America. Surveillance \& Society 10(1): 5-17. Available in http://www.surveillanceand-society.org | ISSN: 1477-7487

Atar, D. (2010). Aportes metodológicos para el estudio de la percepción social de la Ciencia y la Tecnología. Documento de Trabajo, No. 251. Buenos Aires: Universidad de Belgrano.

Bailey, J. y Chabat, J. (eds.) (2002). Transnational Crime and Public Security: Challenges to Mexico and the United States. San Diego: Center for U.S.-Mexican Studies, University of California.

Bressan, G. (2017). Power Mobility and the economic vulnerability of borderlands. Journal of Borderland Studies, vol. 32, No. 3.

Brunet-Jailly, E. (2006). Security and Border Security Policies: Perimeter or Smart Border? A Comparison of the European Union and Canadian-American Border Security Regimes, Journal of Borderland Studies, vol. 21, No.1, Spring.

Cardozo, E. (2010). La politica exterior del gobierno bolivariano y sus implicaciones en el plano doméstico. Caracas: Instituto Latinoamericano de Investigaciones Sociales, Ildis.

Cardozo, E. (2011). El giro de las relaciones entre Venezuela y Colombia: alcances, riesgos y posibilidades. Caracas: Instituto Latinoamericano de Investigaciones Sociales, Ildis.

Carrión, F. y Espín, J. (2011). Relaciones fronterizas: Encuentros y conflictos. Flasco - Ecuador. 
Elhawary, S. (01-06/2008). ¿Caminos violentos a la Paz? Reconsiderando el nexo entre conflicto y desarrollo. Colombia Internacional, 67, pp. 84-100.

Farson, S. (2006). Rethinking the North American Frontier After 9/11, Journal of Borderland Studies, vol. 21, No.1, spring.

Flick, U. (2004). Introducción a la investigación cualitativa. Madrid: Morata.

García Canclini, N. (2000). La globalización: ¿̨productora de culturas híbridas? Ponencia Inaugural del III Congreso Latinoamericano de la Asociación Internacional para el Estudio de la Música Popular - Bogotá [En línea, consultada el 6 de marzo de 2018] http://www.hist.puc.cl/historia/iaspm/ pdf/Garciacanclini.pdf

Glasser, B. y Strauss, A. L. (1967). The discovery of Grounded Theory: Strategies for Qualitative Research. Nueva York: Aldine.

Grimson, A. (Compilador) (2000). Fronteras, nacionese identidades. La periferia como centro. Argentina: Ediciones Ciccus-La Crujía.

González, F. (18/09/2006). El fenómeno político de Álvaro Uribe Vélez. ¿De dónde proviene la legitimidad de este líder elegido por segunda vez como presidente? Instituto de Investigaciones y Debate sobre Gobernanza. Análisis. Disponible en http:// www.institut-gouvernance.org/es/analyse/ficheanalyse- $245 . \mathrm{html}$

González Martínez, M. A. y Betancourt Montoya, M. A. (06/2018). La transformación del Ejército Nacional de Colombia: una interpretación teórica. URVIO, Revista Latinoamericana de Estudios de Seguridad, No. 22, pp. 70-84.

Granada, S.; Restrepo, J. y Vargas, A. (2009). El agotamiento de la política de seguridad: Evolución y transformaciones recientes en el conflicto armado, (pp.27-124), Restrepo, J. Aponte, D. (editores).
Guerra y violencias en Colombia: Herramientas e interpretaciones. Bogotá: Cerac.

Gutiérrez, F.; Wills, M. E. y Sánchez G., G. (2005). Nuestra guerra sin nombre: Transformaciones del conflicto en Colombia. Bogotá: Iepri - Universidad Nacional de Colombia.

Hernández Sampieri, R.; Fernández Collado, C. \& Baptista Lucio, P. (2014). Metodología de la investigación. (6a. ed.). México D.F.: McGraw-Hill.

Hernández, G. y Romero-Arias, C. A. (2020). La Guardia Nacional y la militarización de la seguridad pública en México. URVIO, Revista Latinoamericana de Estudios de Seguridad, No. 25, pp. 87-106.

Human Security Centre. (2005). Human Security report 2005: War and Peace in the $21^{\text {st }}$ Century. New York: Oxford University Press.

International Crisis Group. (ICG). (23/09/2004). Las fronteras de Colombia: el eslabón débil de la política de seguridad de Uribe. Informe sobre América Latina, No. 9.

International Crisis Group. (2011). Annual Report 2011. Disponible en http://www.crisisgroup. org/-/media/Files/Annual\%20Reports/annualreport-2011.pdf

Jenne, N. (2014). Venezuela. La frontera en la agenda de seguridad: los efectos transversales en el caso colombo-venezolano, Dossier de Análisis, No. 03, Universidad de los Andes - Colombia.

Kearney, M. (1998). Transnationalism in California and Mexico at the end of the empire. En Wilson, T. M. and Donnan, H. (Ed.), Border identities. Nation and state at international frontiers, Cambridge: Cambridge University Press.

Keohane, R. and Nye, J. (1987). Power and Interdependence Revisited, International Organization, No. 41, 4, Fall. Boston: Massachusetts Institute of Technology. 
Koff, H (2008). Comparative Border Politics and Structures of Power. Estudios Politicos, No. 32, Jan./ June, Medellín.

Kolossov, V. (2005). Border Studies: Changing Perspectives and Theoretical Approaches, Geopolitics, 10:4, 606-632.

Kolossov, V. y Scott, J. (2013). Selected conceptual issues in border studies. Belgeo. https://doi.org/10.4000/ belgeo.10532.

Martínez, O. (1994). Border People. Life and Society in the US-Mexico borderlands, Tucson: The University of Arizona Press.

Martínez, O. (1997). Border people and their cultural roles: The case of the U.S-Mexican borderlands. En Ganster, P. et al. (Ed.), Borders and Border regions in Europe and North America (pp. 293-298). San Diego: San Diego State University Press.

Monroy, H. M. y Ochoa, A. M. (2009). Análisis de las externalidades negativas de las políticas antidrogas de Colombia sobre las poblaciones fronterizas de Norte de Santander - Táchira. Lecciones para el diseño de políticas públicas. Nova et Vetera. Política Pública y Derechos Humanos, No. 18 (62), pp.9-20.

Morffe P., M. A. (2014). El riesgo percibido sobre la seguridad ciudadana en la Frontera Táchira- Norte de Santander, Trabajo de Grado para optar al Título de Magister Scientiarum en Fronteras e Integración, Universidad de los Andes.

Mouly, C.; Idler, A. and Garrido, B. (2015). Zones of Peace in Colombia’s Borderland, International Journal of Peace Studies, vol. 20, No. 1, summer.

Newman, D. (editor) (1999). Boundaries, Territory and Postmodernity, London: Frank Cass.

Newman, D. (2012). Borders and Conflict Resolution, in Wilson, T. and Donnan, H. (eds.), A Companion to Border Studies, Blackwell, 14, pp. 249-265
Observatorio de Venezuela. (2018). Las migraciones en el contexto colombo-venezolano. Universidad del Rosario y Fundación Konrad Adenauer Stiftung. Disponible en https://www.kas.de/ documents/287914/287963/Las+migraciones+ en + el+contexto+colombo-venezolano.pdf /dee22342-dc38-d405-3ddd-ce94e2d60fe4? $\mathrm{t}=1563801976151$

Observatorio de Venezuela. (2019). El fenómeno migratorio en las zonas de frontera y los retos en seguridad. Universidad del Rosario y Fundación Konrad Adenauer Stiftung. Disponible en https:// migravenezuela.com/web/articulo/migracionvenezolana-en-frontera/1608

Orjuela E., Luis J. (2000). La debilidad del Estado colombiano en tiempos del neoliberalismo y el conflicto armado. Colombia Internacional, No. 49-50, pp. 103-116.

Orozco, G. (2006). El concepto de la seguridad en la teoría de las relaciones internacionales, Revista CIDOB d'Afers Internacionals, No. 72, pp. 161-180.

Paasi, A. (1999). Boundaries as Social Processes: Territoriality in the World Flows, in Newman, D. and Cass, F., Boundaries, Territory and Postmodernity, London.

Páez, P. (2009). Incidencias de la política de seguridad democrática del gobierno de Álvaro Uribe en las relaciones bilaterales con Venezuela. 2002-2008. Monografía presentada como trabajo de grado para optar al grado de internacionalista. Bogotá: Universidad Colegio Mayor de Nuestra Señora del Rosario.

Peña Chivatá, C.; Sierra-Zamora, P. \& Hoyos Rojas, J. (2019). La política de fronteras de Colombia ante las nuevas amenazas de seguridad y defensa. Revista Cientifica General José María Córdova, 17(28), 773-795. http://dx.doi. org/10.21830/19006586.473 
Pizarro L. E. (2003). Colombia. El proyecto de seguridad democrática de Álvaro Uribe, Nueva Sociedad, No. 186, julio-agosto (Coyuntura). Caracas

Ramos G., J. M. y Woo, O. (Coordinadores). (2004). Seguridad Nacional y Fronteriza, México: Universidad de Guadalajara.

Rodríguez M., F. (2015). La gran estrategia de Estados Unidos: en busca de las fuentes doctrinales de las políticas de seguridad internacional de los gobiernos estadounidenses. Colombia Internacional, No. 83, pp. 171-201.

Russell, R. (2003). U.S. Policy toward Latin America: A view from the South. En Roett, R. and Paz, G. (Ed.), Latin America in a Changing Global Environment. Londres: Lynner Rienner Publisher.
Sánchez, F. y Díaz, A. (2007). Geografía de los cultivos ilícitos y conflicto armado en Colombia (pp. 131205). En Sánchez, F. Las cuentas de la violencia. Bogotá: Norma.

Sandin, M. (2013). Investigación cualitativa en educación. Fundamentosy tradiciones. Madrid: Mc Graw and Hill Interamericana.

Strauss, A. \& Corbin, J. (2002). Bases de la investigación cualitativa. Técnicas y procesamientos para desarrollar la teoría fundamentada. Medellín: Universidad de Antioquia.

Tickner, A. y Pardo, R. (2003). Estados Unidos, Colombia y sus vecinos: los desafíos externos de la seguridad democrática. Foreign Affairs en español, octubre-diciembre. 